\title{
COMMUTATIVE FPF RINGS ARISING AS SPLIT-NULL EXTENSIONS
}

\author{
CARL FAITH
}

\begin{abstract}
Let $R=(B, E)$ be the split-null or trivial extension of a faithful module $E$ over a commutative ring $B . R$ is an FPF ring iff the partial quotient ring $B S$ I with respect to the set $S$ of elements of $B$ with zero annihilator in $E$ is canonically the endomorphism ring of $E$, that is $B S^{-1}=\operatorname{End}_{B} E S^{-1}$, every finitely generated ideal with zero annihilator in $E$ is invertible in $B S^{-1}$, and $E=E S^{-1}$ is an injective module over $B$. The proof uses the author's characterization of commutative FPF rings [1] and also the characterization of self-injectivity of a split-null extension [3].
\end{abstract}

Background. A ring $R$ is (right) [F]PF if every [finitely generated] faithful right $R$-module generates the category mod- $R$ of all right $R$-modules. A module $M$ is (Beachy-Blair) cofaithful if there is an embedding $R \rightarrow M^{n}$ for some finite integer $n$. Over a commutative ring $R$, every finitely generated faithful module is manifestly cofaithful (= CF-faithful in [4]). Furthermore, any cofaithful module over a right self-injective ring $R$ generates mod- $R$, since then $R$ splits in any over module, hence $M^{n} \approx R \oplus X$ in mod- $R$ for some $n>0$. This shows that any commutative selfinjective ring $R$ is FPF. Moreover, a commutative FPF ring $R$ is characterized in [1] by the two conditions:

(FPF 1) $R$ is quotient-injective, i.e. the (classical) quotient $\operatorname{ring} Q_{c}(R)$ is injective.

(FPF 2) $R$ is pre-FPF, i.e. finitely generated faithful ideals are generators of mod- $R$ (equivalently, are finitely generated projective $[\mathbf{1}, \mathbf{2}]$ ).

Since any commutative self-injective ring is FPF, it is thereby pre-FPF, that is, $\left(\mathrm{FPF}_{1}\right) \Rightarrow\left(\mathrm{FPF}_{2}\right)$ for injective $R$ : in fact, then $R$ is the only finitely generated faithful ideal.

In [3] we considered the split-null (or trivial) extension $R=(B, E)$ of a faithful $B$-bimodule $E$ over a ring $B$, and characterized the conditions under which $(B, E)$ is right $\mathrm{PF}$ (resp. right self-injective). We say that $E$ is left strongly balanced [3] if $B$ is canonically isomorphic to the endomorphism ring of the right $B$-module $E$; notation: $B=$ End $E_{B}$. Theorem 2 of [3] states

(IN 1) $(B, E)$ is right self-injective iff $E$ is a left strongly balanced injective right $B$-module.

(IN 2) $(B, E)$ is right PF iff $E$ is a left strongly balanced injective right cogenerator over $B$.

Henceforth let $B$ be a commutative ring, and let $E$ be a faithful $B$-module. Then $R=(B, E)$ is commutative, and our main theorem characterizes when $R$ is FPF. To

Received by the editors September 27, 1982.

1980 Mathematics Subject Classification. Primary 16A36, 16A52; Secondary 16A14. 
describe the result, let $S$ denote the multiplicative set of $B$ consisting of all $b \in B$ such that $\operatorname{ker} b=0$, i.e., $b: E \rightarrow E$ is monic. Then, $S$ is a subset of the set $B^{*}$ of regular elements of $B$, hence the quotient ring $Q=B S^{-1}$ embeds in $Q=Q_{\text {c }}(B)$ canonically.

1. Proposition and Definition. An ideal I of $B$ is said to invert or is invertible in a commutative overring $Q$ provided the equivalent conditions hold:

(a) $I^{\prime} I=B$ for $I^{\prime}=(I: B)=\{q \in Q \mid q I \subseteq B\}$.

(b) $\sum_{i=1}^{n} q_{i} I=B$ for finitely many elements $q_{1}, \ldots, q_{n} \in Q$.

(c) There exist elements $q_{i}, \ldots, q_{n} \in Q$, and $b_{i}, \ldots, b_{n} \in I$ so that $q_{i} b_{i} \in B, i=$ $1, \ldots, n$ and $\sum_{i=1}^{n} q_{i} b_{i}=1$.

(d) $I$ is a faithful ideal of $B$, and there exist $q_{i}, \ldots, q_{n} \in Q, b_{i}, \ldots, b_{n} \in I$ so that $q_{i} b_{i} \in B_{i}, i=1, \ldots, n$ and $x=\sum_{i=1}^{n} b_{i} q_{i}(x) \forall x \in I$.

(e) $I$ is a finitely generated faithful projective $B$-module, and if $f \in \operatorname{Hom}_{B}(I, B)$, then $f=q_{s}$, for some $q \in Q$, where $q_{s}(x)=q x \forall x \in B$.

(f) I generates mod- $B$ and if $f \in \operatorname{Hom}_{B}(I, B)$, then $f=q_{s}$ for some $q \in Q$.

Proof. (a) $\Leftrightarrow$ (b) $\Leftrightarrow$ (c) $\Leftrightarrow$ (d) is direct, and (d) $\Leftrightarrow$ (e) uses the dual basis lemma. It can be shown that $b_{1}, \ldots, b_{n}$ generates $I$, that $\left(q_{1}\right)_{s}, \ldots,\left(q_{n}\right)_{s}$ generates $\operatorname{Hom}_{B}(I, B)$, and if $f \in \operatorname{Hom}_{B}(I, B)$, then there exists $c_{i} \in B, i=1, \ldots, n$, so that

$$
f=\sum_{i=1}^{n}\left(q_{i}\right)_{s} c_{i}=\left(\sum_{i=1}^{n} q_{i} c_{i}\right)_{s}
$$

(Necessarily, $c_{i}=f\left(b_{i}\right), i=1, \ldots, n$.)

We say that an ideal $I$ acts faithfully on $E$ if the annihilator $r_{E} I$ of $I$ in $E$ is zero. Since $E$ is faithful, then $B \rightarrow$ End $E_{B}$ canonically, so an ideal $I$ acts faithfully iff $\cap_{b \in I} \operatorname{ker} b=0$ (i.e. when each $b \in B$ is considered as an endomorphism of $E$ ). In this case we say $I$ has zero kernel in $E$. This implies that $I$ is a faithful ideal of $B$ inasmuch as $I c=0$ for some $c \in B$ implies $I c E=0$, and then

$$
r_{E} I=0 \Rightarrow c E=0 \Rightarrow c=0 \text {. }
$$

2. FPF Theorem for SPlit - Null Extensions. Let $E$ be a faithful B-module. Then, $R=(B, E)$ is an FPF ring iff the following three conditions hold:

(2.1) $E$ is injective,

(2.2) $B S^{-1} \approx$ End $_{B} E$ canonically, where $S=\{b \in B \mid \operatorname{ker} b=0\}$,

(2.3) Every finitely generated ideal of $B$ with zero kernel in $E$ is invertible in $B S^{-1}$.

When this is so, then $Q_{c}(R)=\left(B S^{-1}, E\right)$, is self-injective, and $I$ is a projective ideal of $B$.

3. Corollary. If $E$ is a strongly balanced injective module over $B$, then every finitely generated ideal of $B$ acting faithfully on $E$ is projective, so $B$ is pre-FPF.

4. Corollary. If there exists a strongly balanced injective torsion free module $E$ over a domain $B$, then $B$ is FPF, hence Prufer.

Comments. Corollary 3 follows from the theorem, and the characterization in (IN 1) of injective $(B, E)$. Moreover, in Corollary 4 , every finitely generated ideal 
$I \neq 0$ acts faithfully on $E$, hence is projective by the theorem, so $B$ is pre-FPF. Since pre-FPF $\Rightarrow$ FPF $\Leftrightarrow$ Prufer in a domain (see [2]), Corollary 4 follows.

\section{Preliminaries.}

Proof of Theorem 2. We first compute $Q_{c}(R)$. Let $(b, x)=\left(\begin{array}{c}b x \\ o b\end{array}\right)$ denote a typical element of

$$
R=(B, E)=\left\{\left(\begin{array}{ll}
b & x \\
o & b
\end{array}\right) \in\left(\begin{array}{l}
B E \\
O B
\end{array}\right) \mid b \in B, x \in E\right\} .
$$

If also $(c, y) \in R$, then

$$
(b, x)(c, y)=(b c, c x+b y)
$$

so $(b, x)$ is regular in $R$ iff

$$
\operatorname{ker} b=\{y \in E \mid b y=0\}=0 .
$$

This follows since if $b y=0$ and $y \neq 0$, then $(b, x)(0, y)=(0, b y)=0$, so $(b, x)$ is not regular in $R$. Conversely, if we put (1) to 0 , and $(c, y) \neq 0$, then $b c=0$, so $E$ faithful implies $c E \neq 0$, and $0 \neq c E \subseteq \operatorname{ker} b$.

It follows that $S=\{b \in B \mid \operatorname{ker} b=0\}$ is a multiplicative subset of $B^{*}$, so $Q=$ $B S^{-1} \subseteq Q_{c}$. Furthermore,

$$
Q_{c}(R) \subseteq\left(Q, Q \otimes_{B} E\right)=\left(B S^{-1}, E S^{-1}\right)
$$

since if $(b, x) \in R^{*}$, then

$$
(b, x)^{-1}=\left(b^{-1},-b^{-1} x\right) \in\left(B S^{-1}, E S^{-1}\right)=\left(Q, Q \otimes_{B} E\right)
$$

where $b^{-1} x$ is identified with $b^{-1} \otimes x \in Q \otimes_{B} E$. Moreover, every

$$
q=\left(a b^{-1}, t^{-1} y\right) \in\left(B S^{-1}, E S^{-1}\right)
$$

has the form

$$
q=(a t, b y)\left(b^{-1} t^{-1}, 0\right) \in B S^{-1}
$$

that is, $q \in Q_{c}(R)$, proving the inclusion (2) is an equality.

An ideal $K$ of $R$ has the form $K=\left(K_{B}, K_{E}\right)$, for an ideal $K_{B} \subseteq B$ and a $B$-submodule $K_{E}$ of $E$ such that $K_{B} E \subseteq K_{E}$. Since $(b, x)(c, y)=(b c, b y+c x)$ $\forall b \in B, x, y \in E$, then $K$ is faithful in $R$ iff $K_{B}$ acts faithfully on $E$.

An ideal $K$ of $R$ generated by $\left\{\left(a_{\lambda}, x_{\lambda}\right)\right\}_{\lambda \in \Lambda}$ has the form

$$
\begin{aligned}
K & =\left(K_{B}, K_{E}\right)=\sum_{\lambda \in \Lambda}\left(a_{\lambda}, x_{\lambda}\right) R=\left(\sum_{\lambda \in \Lambda} a_{\lambda}, B, \sum_{\lambda \in \Lambda} a_{\lambda} E+\sum_{\lambda \in \Lambda} B x_{\lambda}\right) \\
& =\left(K_{B}, K_{B} E+\sum_{\lambda \in \Lambda} B x_{\lambda}\right)=\left(K_{B}, K_{B} E+K_{E}\right) .
\end{aligned}
$$

\subsection{Proposition. An ideal $K$ (generated as in (5.1)) inverts in}

$$
Q_{c}(R)=\left(B S^{-1}, E S^{-1}\right)
$$

iff $K_{B}$ inverts in $B S^{-1}$ and $x_{\lambda} \in K_{B} E$ for all $\lambda \in \Lambda$. In other words, $K$ is invertible in $R$ iff $K_{B}$ is invertible in $B S^{-1}$ and $K=\left(K_{B}, K_{B} E\right)$. 
Proof. $K$ inverts in $\left(B S^{-1}, E S^{-1}\right)$ iff there exist elements $q_{1}, \ldots, q_{m} \in B S^{-1}$ and $y_{1}, \ldots, y_{m} \in E, m<\infty$, such that $\sum_{i=1}^{n} K\left(q_{i}, y_{i}\right)=R$, equivalently

$$
\sum_{i=1}^{n} q_{i} K_{B}=B
$$

and

$$
E=\sum_{i=1}^{m} y_{i} K_{B}+\sum_{i=1}^{m} q_{i} K_{E}
$$

Furthermore, (3) holds iff

$$
K_{B} \text { inverts in } B S^{-1} \text { and } K_{B}^{-1}=\sum_{i=1}^{m} q_{i} B .
$$

When (5) holds, then (4) is equivalent to

$$
E=\sum_{i=1}^{m} y_{i} K_{B}+K_{B}^{-1} K_{E}
$$

Now by (5.1) (which is a straightforward computation), we have

$$
K_{E}=K_{B} E+\sum_{\lambda \in \Lambda} B x_{\lambda}
$$

and so (6) is equivalent to

$$
E \supseteq \sum_{\lambda \in \Lambda} K_{B}^{-1} x_{\lambda}
$$

or

$$
x_{\lambda} \in K_{B} E \quad \forall \lambda \in \Lambda .
$$

Then, by (5.1), $K=\left(K_{B}, K_{B} E\right)$.

6. Corollary. If $E=E s$ for all $s \in S$, then $K$ inverts in $Q_{c}(R)$ iff $K_{B}$ inverts in $B S^{-1}$.

Proof. The necessity follows from the proposition. Conversely, if $K_{B}$ inverts in $B S^{-1}$, then $K_{B}$ contains an element $s \in S$, so then $E=E s$ implies that $E=K_{B} E$ and so the criterion of the proposition applies.

We can now complete the proof of Theorem 2.

Necessity. If $R=(B, E)$ is FPF, then by (FPF 1$), Q_{c}(R)=\left(B S^{-1}, E S^{-1}\right)$ is self-injective. By (IN 1), then $E S^{-1}$ is a strongly balanced injective $B S^{-1}$-module. By [1], $R$ is integrally closed in $Q_{c}(R)$, hence contains all nilpotents of $\left(B S^{-1}, E S^{-1}\right)$, so $R \supseteq\left(0, E S^{-1}\right)$, that is,

$$
E=E S^{-1} \text {. }
$$

Since $E$ is thereby injective over $B S^{-1}$, then (2.1) holds via flatness of $B S^{-1}$ over $B$. Since $E S^{-1}$ is strongly balanced over $B S^{-1}$, then (2.2) holds.

Finally, if $I=\sum_{i=1} b_{i} B$ acts faithfully on $E$, and $F=\sum_{i=1}^{n} b_{i} E$, then

$$
K=(I, F)=\sum_{i=1}^{n}\left(b_{i}, 0\right) R
$$


is a finitely generated ideal of $R$ and faithful, so $R$ FPF implies via Proposition 1 that $K$ inverts in $Q_{c}(R)$, hence $I=K_{B}$ inverts in $B S^{-1}$ by Proposition 5. This yields (2.3).

Sufficiency. (2.1) implies that $E$ is divisible, hence $E$ is canonically a $B S^{-1}$-module. Since $B S^{-1}$ is a ring epic of $B$, then $E$ is also injective over $B S^{-1}$. Now (2.2) evidently implies $B S^{-1}=\operatorname{End}_{B S^{-1}} E$ canonically, so $Q_{c}(R)=\left(B S^{-1}, E S^{-1}\right)$ is self-injective by (IN 1), so $R$ is (FPF 1).

It remains to show that $R$ is (FPF 2). Let $K=\left(K_{B}, K_{E}\right)$ be a finitely generated faithful ideal of $R$. Then $K_{B}$ acts faithfully on $E$, as shown supra Proposition 5. Since $K_{B}$ is finitely generated in $B$, then $K_{B}$ is invertible by (2.3), so $K$ is invertible by Corollary 6.

ACKNOWLEDGEMENT. I wish to acknowledge the referee for suggestions that improved the exposition.

Problems. 1. Characterize when $R=(B, E)$ is FPF for a general faithful bimodule $E$ over a noncommutative ring. Unfortunately, arbitrary noncommutative FPF rings have yet to be characterized, although the list of characterized FPF rings includes (1) semiprime, (2) prime, (3) self-injective, (4) Noetherian semiprime, (5) semiperfect Noetherian etc. (see [6]).

2. Characterize $F P^{2} F$ split-null extensions. Even for commutative $R$ this is open, essentially since commutative $\mathrm{FP}^{2} \mathrm{~F}$ rings have not been characterized: Here is a “working” conjecture: A commutative ring $R$ is $\mathrm{FP}^{2} \mathrm{~F}$ iff $R$ is pre-FP ${ }^{2} \mathrm{~F}$ (i.e. finitely presented faithful ideals are projective) and $Q_{c}(R)$ is FP-injective. The sufficiency of these conditions follows as in the proof of the corresponding conditions (FPF 1 and 2 ) in [1]. (Hint: an FP-injective ring splits in any finitely-presented over-module.)

3. If $B=\operatorname{End}_{B} E$ and $E$ is injective over $B$, is $B$ necessarily FPF? The answer is yes when $B$ is of Noetherian domain, since then the problem may be reduced to the case where $E$ is indecomposable, hence then $B=\operatorname{End}_{B} E$ is the ring of $p$-adics (where $P$ is the prime in Ass $E$; see [3] and [5], Chapter 11). Thus, in this case $B$ is a discrete valuation domain hence a PID, so a fortiori FPF.

\section{REFERENCES}

1. C. Faith, Injective quotient rings of commutative rings. II, Injective Modules and Injective Quotient Rings, Lecture Notes in Pure and Appl. Math., vol. 72, Dekker, New York, 1982.

2. __ Injective quotient rings of commutative rings. I, Module Theory, Lecture Notes in Math., vol. 700 (C. Faith and S. Wiegand, eds.), Springer-Verlag, Berlin and New York, 1979.

3. __ Self-injective rings, Proc. Amer. Math. Soc. 77 (1979), 157-164.

4. __ Algebra II: Ring theory, Springer-Verlag, Berlin and New York, 1976.

5. _ Injective modules over Levitzki rings, Injective Modules and Injective Quotient Rings, Lecture Notes in Pure and Appl. Math., vol. 72, Dekker, New York, 1982.

6. C. Faith and S. Page, FPF ring theory: faithful modules and generators of Mod- $R$, Lecture Notes of the London Math. Soc., Cambridge Univ. Press, 1984.

Department of Mathematics, Rutgers University, Hill Center for the Mathematical ScienCes, Busch Campus, New Brunswick, New Jersey 08903 